\title{
Disseminated Blastomycosis: A Case Report
}

\section{Tadi LJ ${ }^{*}$, Uma B ${ }^{2}$, Ariyanachi $\mathrm{K}^{3}$ and Reddy $\mathrm{NC}^{4}$}

${ }^{1}$ Department of Microbiology, AIIMS Bibinagar, Yadadri-Bhuvanagiri, India

${ }^{2}$ Department of Microbiology, NIMS, Hyderabad, India

${ }^{3}$ Department of Anatomy, AIIMS Bibinagar, Yadadri-Bhuvanagiri, India

${ }^{4}$ Department of Medicine, Mediciti Institute of Medical Sciences, Medchal, India

${ }^{*}$ Corresponding author: Tadi LJ, Additional Professor, Department of Microbiology, AIIMS Bibinagar, Yadadri-Bhuvanagiri Dist., HMDC 508126, India, Tel: +91-7702985555, E-mail: drlakshmijyothitadi@gmail.com , lakshmi.micro@aiimsbibinagar.edu.in

Citation: Tadi LJ, Uma B, Ariyanachi K and Reddy NC (2021) Disseminated Blastomycosis: A Case Report. J Case Rep Stud 9(2): 204

Received Date: November 09, 2021 Accepted Date: December 14, 2021 Published Date: December 16, 2021

\begin{abstract}
Blastomycosis is known for its propensity to mimic other illnesses, which often leads to incorrect or delayed treatment. Here we present a case report of a patient working in Wisconsin who was misdiagnosed as Tuberculosis since he was an Indian. The patient was not evaluated on endemic grounds for Blastomyces where he was working for couple of years. After he developed skin lesions along with increased shortness of breath, lesions wereevaluated which showed large yeast cells resembling Blastomyces. The treating pulmonologist evaluated his occupational history and was revealed with endemicity for Blastomyces.
\end{abstract}

Keywords: Blastomyces Dermatitidis; Endemic Area; Bilateral Non-Resolving Pneumonia; AntiTuberculous Treatment; Ct-Guided Trucut Biopsy; Liposomal Amphotericin B 


\section{Introduction}

Blastomyces dermatitidis is the etiologic agent of blastomycosis, also called North Americanblastomycosis. It is the imperfect stage (asexual form) of Ajellomyces dermatitidis.

Typically, these infections occur after inhalation of airborne conidia, although in some casescutaneous inoculation of the fungus occurs $[1,2]$. The clinical manifestations of these infections are highly dependent on the immune status of the host. The sexual form is heterothallic and requires opposite mating types for fertile cultures. Infection occurs with equal frequency with both mating types. At $25-30{ }^{\circ} \mathrm{C}$, the asexual form initially produces a fluffy white colony on routine mycologic medium. Some strains develop tan, glabrous colonies without conidia, and others produce light brown colonies with concentric rings [1]. On primary isolation, colonies appear in 1-3 weeks. The mould form of B. dermatitidis produces $2-10 \mu \mathrm{m}$, spherical, ovoid, or pyriform conidia located on aerial hyphae and lateralconidiophores. Thick-walled chlamydoconidium 7-18 $\mu \mathrm{m}$ in diameter may be observed in older cultures. The colony and conidia resemble those of Chrysosporium spp. and may be indistinguishable from an early culture of Histoplasma capsulatum, having only hyphae, conidiophores, and microconidia. In mammalian tissues, broad-based budding yeasts with thick refractile walls varying in diameter from 8 to $30 \mu \mathrm{m}$ are observed. The yeast form can also be produced in vitro at $37^{\circ} \mathrm{C}$ on blood agar, inhibitory mould agar or brain-heart infusion(BHI) agar. The colony of the yeast phase is usually cream, with a granular surface. Clinical manifestations- pulmonary disease ( $<50 \%$ of infected individuals) Extrapulmonary: skin, bone, genitourinary tract, CNS (disseminated disease more common in immunosuppressedpatients) [3].

\section{Case report}

On Dec 15TH 2011 at Mediciti Hospitals (Citi centre)- A 29-year-old male patient came withcomplaints of multiple swellings over left lower limb, right arm, thigh \& anterior abdominal wall for 15 days. Cough of long-term history of over 6 months which was irritable productive cough with haemoptysis associated discomfort in the chest, along with Shortness of breath, nausea \&vomiting (non-projectile). Before admission patient was a follow-up case of Bilateral non-resolving pneumonia of lowerlobe with right sided pneumonic effusion on modified Anti Tuberculous treatment (ATT) since 2 months \& 19 days. His respiratory illness started 6 months back \& was evaluated extensively outside with ChestX ray, C T Chest, T B biopsy, CT-guided Trucut biopsy. His biopsy reports were suggestive of inflammatory pathology with langerhan's cells. On this basis and him being an Indian citizen he was started on 4 drugs anti Tuberculous treatment (ATT) outside after 10 days. Hetook ATT for 2 months but there was no improvement. He visited OPD in Nov 2011 with cough and fever, he underwent Thoracocentesis. Pleural fluid analysis was exudative in nature with lymphocytic predominance along with derangedliver parameters. He was admitted on Dec 15th, 2011. On examination his Respiratory system findings - decrease intensity of breath sounds bilaterally in mammary area, infra mammary area, axillary area, infra-axillary area, infra scapular area and lower inter scapular area with bilateral basal early inspiratory coarse crepts. Cardiovascular system, central nervous system - normalLaboratory findings (Figure 1). 


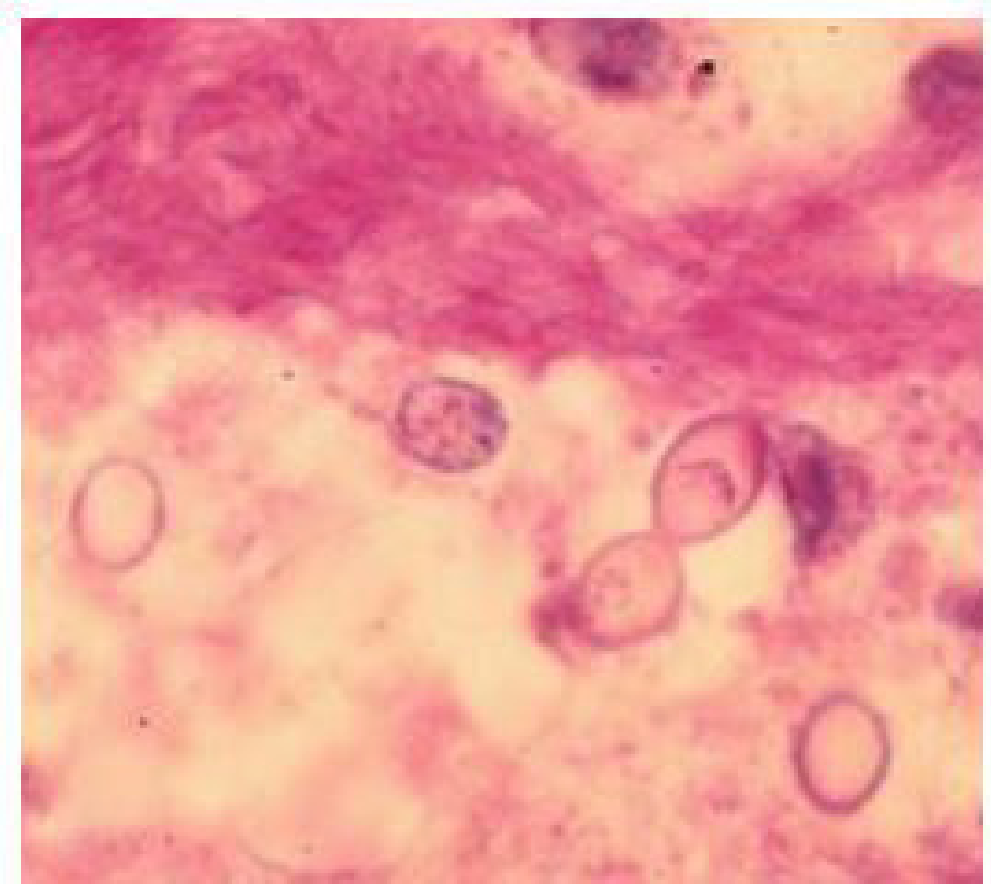

Figure 1: From tissue section- Broad-based budding yeasts of Blastomyces dermatitidis

Serum electrolytes - normal initially from day 5 onwards serum sodium \& serum chlorides were decreasing, CBP first 48 hours was normal, after 72 hours there was gradual increase inthe WBC count from 8000 Cumm to 24000 Cumm (Figure 2).

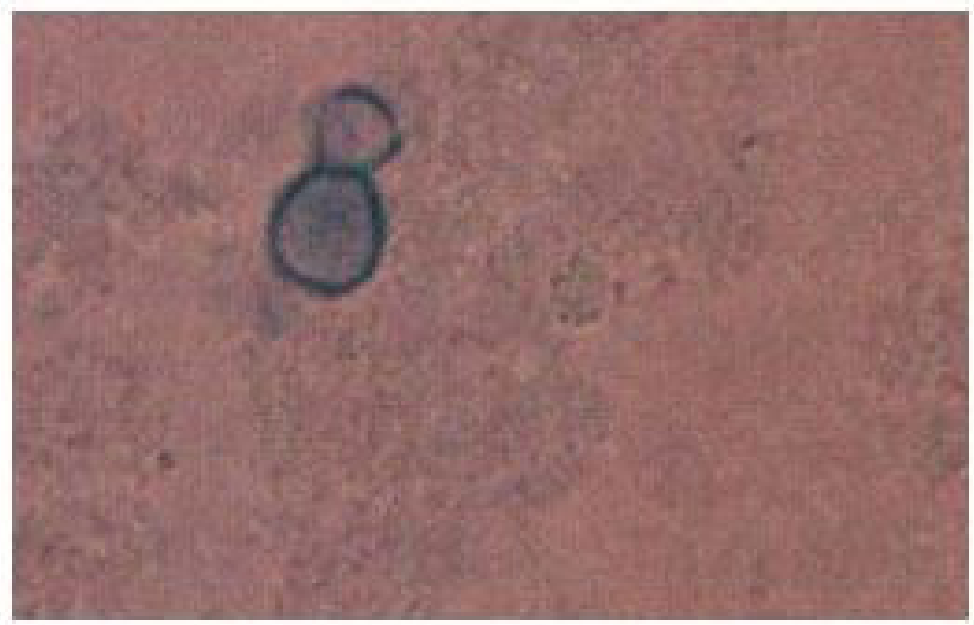

Broad based budding and thickened cell walls and globose shape

Figure 2: Yeast form from the brownish blood-tinged pus sample collected from the right thigh 
Viral parameters were negative. Liver function tests showed increase in SGOT \& SGPT levels. With careful monitoring he was started with modified ATT which had Streptomycin+MACOXPLUS (Rifampicin \& Isoniazid) +OFLOMAC (ofloxacin) +COMBUTOL(Ethambutol) (Figure 3).

On 18th December 2011 he was discharged in clinically stable condition \& was on regularfollow-up on OPD basis. As his liver parameters were deranged his ATT was further modified \& MACOXPLUS (Rifampicin \& Isoniazid) was stopped \& continued other ATTdrugs (Figure 4).
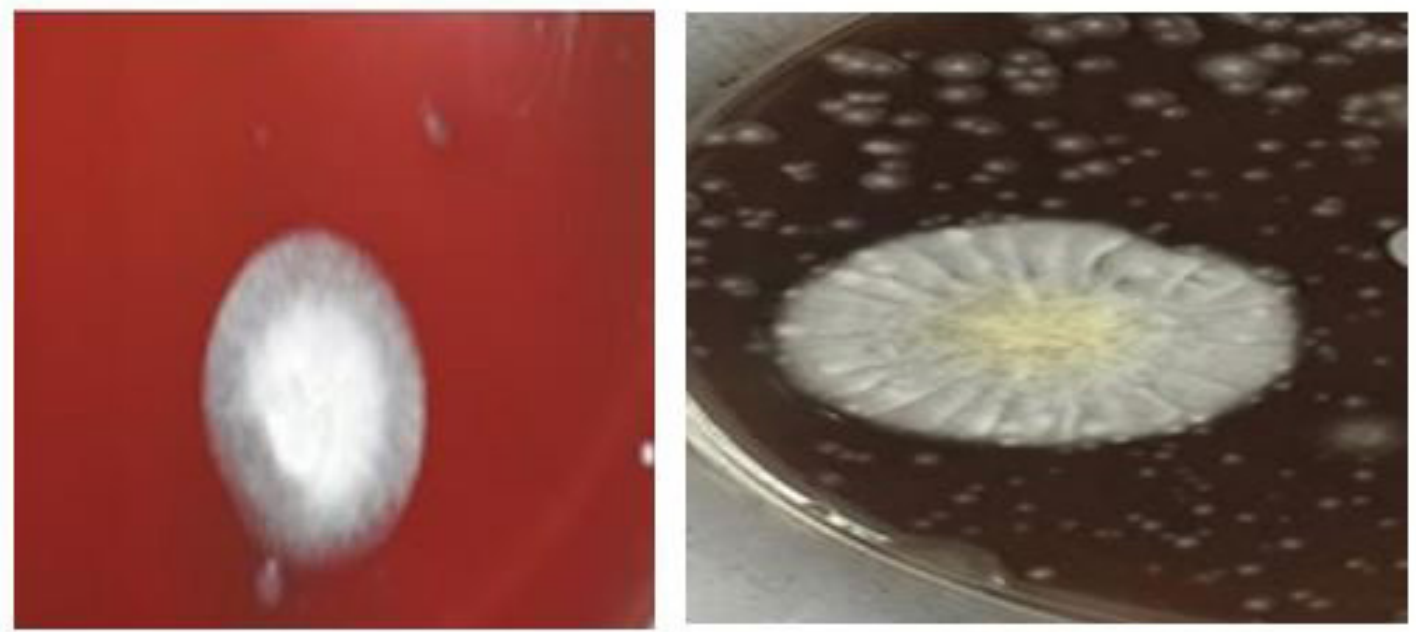

Figures 3 and 4: Culture plate after 24 hours \& 48 hours showing buff coloured velvetymould form

After discharge 1 week later, he got admitted as he developed multiple subcutaneous swellings over posterior aspect of right arm, lateral side of left thigh and over anterior abdomen. Swellings were approximately $3 \times 2 \mathrm{~cm}$ in size, painful, mobile, non-adherent to skin. He complained of Cough with minimal expectorant associated with haemoptysis alongwith vomitings 2 to 3 episodes / day which was non-projectile and non-bilious in nature. Healso complained of loss of weight of nearly $8-12 \mathrm{~kg}$ in the last 3 months.

Patient was diagnosed with left sided pneumothorax with ICD in situ, bilateral lower lobepneumonitis with cellulitis over right lower limb.

\section{Important findings}

\section{Chest X-ray}

Chest $\mathrm{x}$-ray revealed right sided pneumothorax, ICD was placed, and repeated $\mathrm{x}$-rays were showing complete expansion and subsequently pleurodesis with Tetracycline was done following which his shortness of breath decreased.

Meanwhile pustular lesion on the thigh ruptured oozing with pus. Pus sample was sent forMicroscopy, Culture identification for bacterial and fungal growth.

\section{Cytology}

Bits of tissue scraped along the edges of the ulcer were sent for histopathological examination. 


\section{On Microscopy}

Wet mount picture showed large broad based yeast cells and some like figure eight, based onthese findings bits of tissue were sent for histopathology.

Provisionally diagnosing it as Blastomyces, we informed the treating Pulmonologistregarding these findings.

History from the patient was revealed regarding his occupation at Wisconsin, one of theknown endemic areas for Blastomyces dermatitidis.

Meanwhile patient was started on Inj Amphotericin B $1 \mathrm{mg} / \mathrm{kg}$ body weight as slow infusion.

Histopathology sections the next day at our center also has confirmed Blastomyces and wassent for second opinion.

Confirmation with other NABL accredited lab-H \& E stain of lung tissue Core of lung tissue focally lined by ciliated columnar epithelium. The sub epithelium shows non-necrotizing epithelioid cell granulomas with centralsuppuration. Many Langhans giant cells are seen but caseous necrosis is not apparent. In addition, fewrounded nucleated fungal organisms with thick refractive wall are seen within the granulomas. Focally broad-based budding of organisms also seen.

\section{Impression}

Suppurative granulomatous inflammation to fungal organisms consistent with Blast mycosis. Meanwhile fungal culture on Sabouraud dextrose aga rwith antibiotics, sheep blood agar which was processed in BSL-2 CLASS 2 B2 cabinet and was incubated at $37^{\circ} \mathrm{C}$ and at roomtemperature. The mycelial form of $\mathrm{B}$. dermatitidis is non-diagnostic and conversion to the yeast at $37^{\circ} \mathrm{C}$ is necessary for definitive identification. After 36 hours of incubation showed Colonies varying from membranous yeast-like colonies to cottony white to buff colored withno pigmentation on the obverse mould-like colonies at $25^{\circ} \mathrm{C}$. When grown at $37^{\circ} \mathrm{C}$ yeast phase colonies showed wrinkled, folded, and glabrous creamy white colonies.

Microscopy from the yeast culture showed oval yeast with thick double-contoured cell wallsand broad-based budding.

Routine Microscopy, Histopathology, Culture confirmed Blastomyces dermatitis. Sample andculture slants were sent to Mycology division PGIMER (ID number - NCCPF\#240006) where it was confirmed the same.

Chemical pleurodesis was done with removal of ICD, patient developed dyselectrolytemia,and diagnosed as invasive Blastomycosis with type-1 respiratory failure with right sided pleurodesis status, he was shifted to isolation room with critical monitoring. Treatment switched to Liposomal Amphotericin B $100 \mathrm{mg} / \mathrm{OD}$.

Day 4 of the treatment, patient was stable, not drowsy, no shortness of breath.He was discharged on day $15^{\text {th }}$ with all the lab parameters normal.

\section{Results and Discussion}

B dermatitidis is notoriously difficult to separate from its natural habitat. Only a few cases ofeffective isolation have been documented, and even fewer have been linked to disease outbreaks. Blastomycosis can have a variety of clinical symptoms, ranging from asymptomatic illness to a full-blown infection. 
Our patient revealed earlier occupation at Wisconsin. Bruce et al, and Reed et al, conclude that B. dermatitidis in the soil can be a reservoir for human infection and also has shown thatin Wisconsin, blastomycosis in humans has been a reportable disease since $1985[2,3]$.

Patient presented earlier only with respiratory symptoms in the form of non-resolving irritable dry cough for more than 4 months following which he was on anti-tuberculosis treatment. Then he shifted from Wisconsin to Chicago. His occupational history was not evaluated and he, being an Indian with an unresolving cough despite antibiotic therapy alongwith anti-tussive was evaluated for Tuberculosis. Non-specifically based on lung shadows inthe lower lobe of both lungs he was started on Anti-tuberculosis treatment.

Blastomycosis is known for its propensity to mimic other illnesses, which often leads to incorrect or delayed treatment. Fever, chills, and cough are symptoms of clinically acute blastomycosis, which are similar to those of bacterial pneumonia. Treatment for suspected community-acquired pneumonia is frequently started, and a sputum or tissue culture is collected only after the patient fails to react to treatment. Chronic pulmonary symptoms are more common than acute pulmonary symptoms. Patients have low-grade fevers that come and go, a moderate chronic productive cough, chest discomfort, and haemoptysis. Symptomssuch as malaise, tiredness, and weight loss are also common. Tuberculosis and atypical pneumonia have been misdiagnosed as a result of these symptoms [4].

Anil kumar et al., reported a similar case where a 32-year-old man who returned to India aftervisiting Chicago, Illinois, and was treated for TB for 12 months before being diagnosed with blastomycosis [5]. Matthew et al., also reported a similar case where 25 year old male from Mississippi river valley with complaints of haemoptysis, fatigue, weight loss, and fever and CT chest showing a large cavitary lesion in the right upper lobe. Based on the suspicion of TB, the patient was started on empirical Anti-Tuberculosis Treatment for 3 months but later based on the fungal culture report of right upper lobe lesion, he was diagnosed to be infected with Blastomyces dermatitidis [6].

On reporting to Mediciti hospital OPD after analysing completely his previous X ray, C T Chest, T B biopsy, CT-guided Trucut biopsy, he was subjected to thoracocentesis after completely evaluating his past history of illness. Pleural fluid analysis was exudative in nature with lymphocytic predominance. Pleural illness has been described as "very uncommon" with this infection; nevertheless, in two studies, 26 percent and 42 percent of patients with blastomycosis exhibited signs of pleural effusion, respectively. In conclusion, none of the radiographic patterns can be used to diagnose a fungal infection [7,8]. Failla et al., presented a similar case series where 2 out of 7 patients with pulmonary blastomycosis thoracentesis, which yielded markedly elevated pleural fluid total protein [9].

Many blastomycosis patients with radiographic mass lesions are initially misdiagnosed as having lung cancer. Almazthoub et al., presented a similar case where a 51-year-old residentof Ohio river basin presented with symptoms of unproductive cough, mild dyspnoea, unexplained weight loss with CT chest demonstrating right upper lung lobe mass with hilar and mediastinal lymphadenopathy and pericardial and pleural effusion. Initially the case wassuspected to be malignancy but the aspiration of lymph nodes and subsequent pathological examination large spherical yeast cells of consistent size showing broad based buds with multinucleated giant cells. GMS stain showed fungal elements indicating of Blastomyces dermatitidis [10]. None of the radiographic patterns can be used to diagnose a fungal infection.

After thoracocentesis, our patient was admitted and as his liver parameters were deranged, rifampicin and isoniazid was stopped and alternative ATT drugs were given. He was discharged after 3 days when his condition was stabilised. After discharge 1 week later, he got admitted as he developed multiple subcutaneous swellings over posterior aspect of rightarm, lateral side of left thigh and over anterior abdomen and dyspnoea and haemoptysis.

Swellings were approximately $3 \times 2 \mathrm{~cm}$ in size, painful, mobile, non-adherent to skin and puswas oozing out from the swellings. Pus sample was sent for Microscopy, Culture identification for bacterial \& fungal growth Bits of tissue scraped along the edges of the ulcerwere sent for histopathological examination. Wet mount picture showed large broad based yeast cells and some like figure 
eight, based on these findings bits of tissue were sent for histopathology. Based on the occupational history and histopathological confirmation, a diagnosis of Blastomyces dermatitidis was made. Patient was started on Inj Amphotericin B 1mg/ kg body weight as slow infusion. CT chest showed right sided pneumothorax and was treated with ICD was placed, and repeated x-rays were showing complete expansion and subsequently chemical pleurodesis was done with removal of ICD, patient developed dyselectrolytemia, and diagnosed as invasive Blastomycosis with type-1 respiratory failure Treatment switched to Liposomal Amphotericin B 100 mg /OD. Day 4 of the treatment, patient was stable, not drowsy, no shortness of breath. He was discharged on day $15^{\text {th }}$ with all the lab parameters normal. Dissemination from the lungs is not unusual and can involve any organ. Blastomycosis can have a fulminant course, with fevers, chills, and shortness of breathin a small number of patients. Within one-week, rapid systemic spread and development to acute respiratory distress syndrome occurs, often resulting in death [11].

Following pneumonia, the most frequent symptom of blastomycosis is cutaneous lesions. The lesions could be ulcerative or verrucous. The verrucous type features a raised, uneven border with crusting and drainage over a subcutaneous abscess. These lesions have papillomatosis, epidermis downward growth with intraepidermal abscesses, and inflammatory cells in the dermis on histology. Cutaneous ulcers caused by blastomycosis have sharp, heaped-up edges and frequently include exudate at the base. These blastomycosis ulcers develop from subcutaneous pustular lesions that discharge spontaneously. Micro abscesses are commonly seen on biopsy, even in individuals who have minimal clinically visible inflammation in the ulcer. Without with, subcutaneous localization is possible. The presence of skin lesions makes it easier to diagnose blastomycosis [12].

The median duration from beginning to diagnosis in blastomycosis-endemic areas like Illinois is 128 days (range 12-489 days) [13]. The patient in this case had worked for 6 months in an area with a high prevalence of B. dermatitidis. The presence of skin lesions, negative mycobacterial cultures, and the patient's lack of response to anti-TB therapy, shouldhave highlighted the possibility of blastomycosis.

Pneumonia and cutaneous ulcers or verrucous skin lesions are typical symptoms of blastomycosis, although diagnosis is often delayed. In non-disease-endemic locations wheretuberculosis is common, a high index of suspicion is required to identify blastomycosis.

Patients who do not react to anti-TB therapy should provide a detailed travel history to clinicians.

\section{Conclusions}

Specific treatment was very much delayed until he got admitted in our hospital. Since he wasan Indian, he was started on Anti tuberculous treatment. After he developed skin lesions along with increased shortness of breath, lesions showed large yeast cells. The patient was not evaluated on endemic grounds for Blastomyces where he was working for couple of years. Occupational history of exposure to Blastomyces dermatitidis was reported earlier.

Only after his return to his hometown in India, the treating pulmonologist evaluated his occupational history and was revealed with endemicity for Blastomyces after he developedthe skin lesions. Simple oral antifungal like Itraconazole, Voriconazole could have helped him for early recovery without massive weight loss caused by Disseminated Blastomycosisdue to delay in proper diagnosis and treatment. 


\section{References}

1. McBride JA, Gauthier GM, Klein BS (2017) Clinical Manifestations and Treatment of Blastomycosis. Clinics in chest medicine 38: 10.1016/j.ccm.2017.04.006.

2. Klein BS, Vergeront JM, Weeks RJ, Nanda Kumar U, Mathai G, et al. (1986) Isolation of Blastomyces dermatitidis in Soil Associated with a Large Outbreak of Blastomycosis in Wisconsin. University of Wisconsin-Madison Clinical Sciences Center: New England Journal of Medicine 10.1056/NEJM198602273140901.

3. Reed KD, Meece JK, Archer JR, Peterson AT (2008) Ecologic Niche Modeling of Blastomyces dermatitidis in Wisconsin. PLoS ONE 3: e2034.

4. Kuzo RS, Goodman LR (1996) Blastomycosis. Semin Roentgenol 31: 45-51.

5. Kumar A, Kunoor A, Eapen M, Singh PK, Chowdhary A (2019) Blastomycosis Misdiagnosed as Tuberculosis, India. Emerg Infect Dis 25: 1776-7.

6. Koroscil MT, Andrew S (2018) Chronic Pulmonary Blastomycosis Mimicking Pulmonary Tuberculosis. Military Medicine 183: e332-e333.

7. Bradsher RW, Rice DC, Abernathy RS (1985) Ketoconazole therapy of endemic blastomycosis. Ann Intern Med 103: 872-9.

8. Kinasewitz GT, Penn RL, George RB (1984) The spectrum and significance of pleural disease in blastomycosis. Chest 86: 580-4.

9. Failla PJ, Cerise FP, Karam GH, Summer WR (1995) Blastomycosis: pulmonary and pleural manifestations. South Med J 88: 40510.

10. Almajthoub Z, AlNabulsi M, Watanabe Tejada LC, Go L (2019) Blastomycosis Causing an Uncommon Presentation of Thoracic Lymphadenopathy, Pleural Effusions, and Pericardial Effusion. American Journal of Respiratory and Critical Care Medicine 199: A6879.

11. Fang W (2007) Imaging Manifestations of Blastomycosis: A Pulmonary Infection with Potential Dissemination. RadioGraphics 27: 641-55.

12. Saccente M, Woods GL (2010) Clinical and laboratory update on blastomycosis. Clin Microbiol Rev 23: 367-81.

13. Dworkin MS, Duckro AN, Proia L, Semel JD, Huhn G (2005) The epidemiology of blastomycosis in Illinois and factors associated with death. Clin Infect Dis 41: e107-11. 


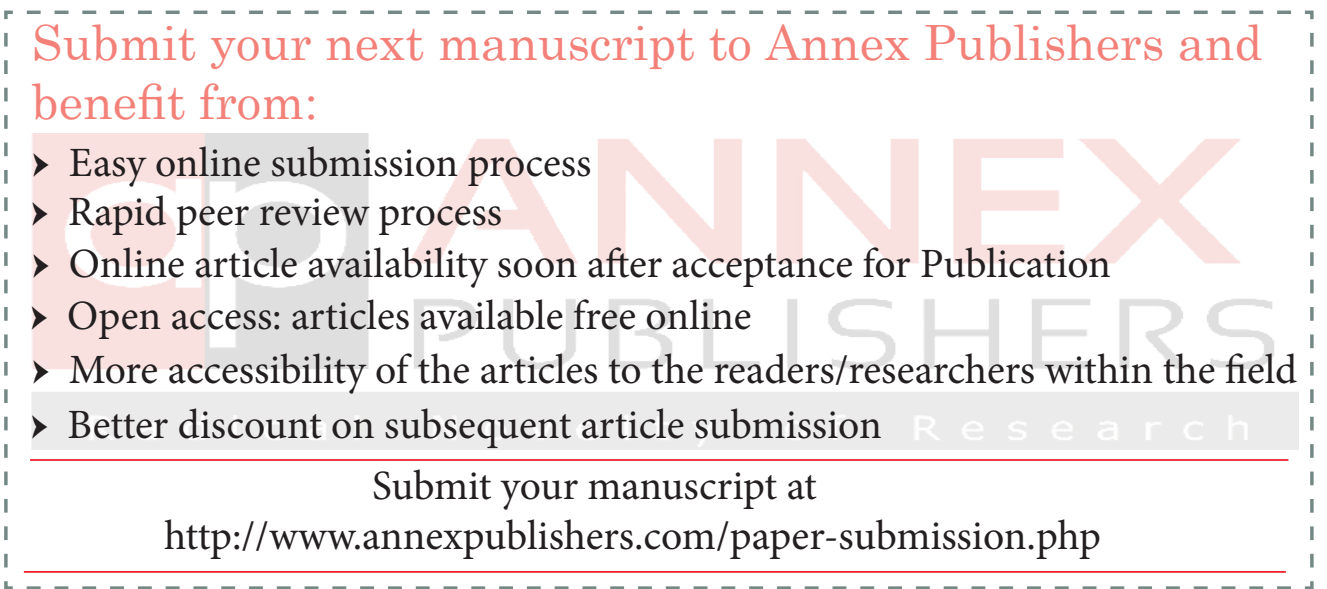

